\title{
Expression of peripheral monocytic programmed death ligand-1 in severe sepsis combined with HBV-related cirrhosis. A pilot observational study
}

\author{
YI LU, GUOXING WANG, CHUNSHENG LI
}

Department of Emergency Medicine, Beijing Friendship Hospital, Capital Medical University, China

\begin{abstract}
Introduction: Hepatitis B virus (HBV)-related liver cirrhosis $(L C)$ complicated with severe sepsis (SS) leads to HBV-related acute-on-chronic liver failure (HBV-ACLF). Programmed cell death ligand-1 (PD-L1)-associated immunosuppression is involved in both LC and SS. This study aimed to examine the expression and clinical relevance of $P D-L 1$ on peripheral CD14+ monocytes in sepsis-induced $H B V-A C L F$.

Material and methods: PD-L1 expression on peripheral CD14+ monocytes among the healthy control (HC), LC and LC + SS groups was examined using flow cytometry analysis and compared. In the LC + SS group, an SS-induced ACLF subgroup was identified. LC + SS patients were followed up for 28 days. The correlations between monocytic PD-L1 expression and illness severity scores and the prognostic value of monocytic PD-L1 expression in SS-induced HBV-ACLF patients was examined.

Results: There were 17, 30 and 70 participants in the HC, LC and LC +SS groups, respectively. The monocytic PD-L1 expression was higher in the LC group compared with the HC group and in the LC + $S S$ group compared with the LC group. The monocytic PD-L1 expression was positively correlated with the illness severity scores in LC + SS patients and predicted 28-day mortality of SS-induced HBV-ACLF patients $(n=59)$.

Conclusions: Severe sepsis exhibits a superimposed effect of monocytic PD-L1 up-regulation on the basis of liver cirrhosis, and monocytic PD-L1 expression predicts 28-day mortality of SS-induced $H B V-A C L F$.
\end{abstract}

Key words: PD-L1, ACLF, sepsis, immunosuppression, liver cirrhosis.

(Cent Eur J Immunol 2021; 46 (2): 217-224)

\section{Introduction}

Severe bacterial infection represents the most common extra-hepatic cause of mortality of liver cirrhosis [1], and also is a sign of acute decompensation (AD) of liver cirrhosis [2]. In China, hepatitis B virus (HBV) infection remains the most common cause of liver cirrhosis [3]. The complication of severe sepsis (SS) with HBV-related liver cirrhosis (LC) always lead to AD and organ dysfunction, which was defined as acute-on-chronic liver failure (ACLF) [1, 2]. The development of ACLF is associated with significantly increased short-term and long-term mortality compared with stable chronic liver disease [4].

Immunosuppression plays a key role in the pathogenesis of both SS and LC [5]. Monocytes are involved in the development and progression of cirrhosis $[6,7]$ and sepsis [8]. T cell exhaustion has been shown in both immunosuppressive conditions. Over-expression of programmed cell death-1 (PD-1) on T cells is the most prominent feature of $\mathrm{T}$ cell exhaustion [9]. The functioning of PD-1 relies on its engagement with programmed cell death ligand-1 (PD-L1) and programmed cell death ligand-2 (PD-L2) [10]. PD-L1 is mainly expressed on T cells, B cells, dendritic cells (DCs) and macrophages and functions in the peripheral tissues, while PD-L2 is primarily expressed on antigen presenting cells (APCs) and functions predominantly in lymphoid organs [11]. The binding of monocytic PD-L1 with lymphocytic PD-1 triggers second signals to negatively modulate T cell activation [12]. Also, PD-L1 also exerts immunosuppressive functions by up-regulating the suppressive effect of regulatory $\mathrm{T}$ cells (Tregs) and directly inhibiting monocyte functions $[13,14]$. Anti-PD-L1 therapy partially reversed monocyte-related immunosuppression [14].

Monocytic PD-L1 expression is increased in both sepsis [15] and liver cirrhosis [16]. In septic shock patient,

Correspondence: Chunsheng Li, MD, Department of Emergency Medicine, Beijing Friendship Hospital, Capital Medical University, No. 95, Yongan Rd., Xicheng District, Beijing, postalcode 100050, China, e-mail: lcscyyy@163.com Submitted: 30.10.2019; Accepted: 16.10.2020 
monocytic PD-L1 over-expression was related to 28-day mortality [15], and anti-PD-L1 therapy significantly reversed monocytic dysfunction and inhibited T cell apoptosis in cecal ligation and a puncture (CLP) sepsis model and further improved survival [14]. In HBV-ACLF models, PD-L1 up-regulation in the liver sample sections was established as a marker of HBV-ACLF $[15,17]$.

However, whether sepsis would bring additional monocytic-related immunosuppression to cirrhotic patients and whether monocytic PD-L1 overexpression is of prognostic value for HBV-ACLF patients is not clear. Thus, we examined PD-L1 expression on CD14+ monocytes in LC + SS patients compared with LC patients, as well as the prognostic value of monocytic PD-L1 expression in HBV-ACLF patients.

\section{Material and methods}

\section{Ethical statement}

The study protocol was conducted in line with the Helsinki Declaration of 1975, as revised in 2000. Written informed consent was waived because the blood samples in this study were obtained from residual blood samples which were collected for routine clinical use. The study was approved by the Beijing Chao-yang Hospital ethics committee.

\section{Participants enrollment}

All the participants were $\geq 18$ years old and were divided into the healthy control (HC) group, the LC group and the LC + SS group. The LC group and the LC + SS group patients were enrolled from the Emergency Department of Beijing Di-tan Hospital. Age- and sex-matched healthy controls without obvious infection during the past 3 months were recruited.

According to the medical records from the Beijing Di-tan Hospital, all the patients enrolled in the LC group had a history of persistent HBV infection for more than 6 months. Liver cirrhosis was diagnosed previously according to the past medical history, clinical manifestations and laboratory/auxiliary examination results and presented with or had a history of at least one of the following complications of decompensated liver cirrhosis: ascites, esophagogastric variceal hemorrhage and hepatic encephalopathy.

SS was diagnosed according to the 2012 Surviving Sepsis Campaign guideline [18]. LC + SS group patients should meet the diagnostic criteria of both LC and SS. The diagnosis of ACLF relies on two mandatory conditions: LC with acute decompensation and organ dysfunction. Acute decompensation patients were complicated with at least one of the following acutely developed complications: ascites, hepatic encephalopathy, gastrointestinal hemorrhage and bacterial infection [2]. According to the $\mathrm{AD}$ definition, all the $\mathrm{LC}+\mathrm{SS}$ patients met the AD crite- ria. The diagnosis of organ dysfunction was in accordance with the Chronic Liver Failure-Sequential Organ Failure Assessment (CLIF-SOFA) grading system [2]. LC + SS patients with CLIF-SOFA grade 0 and CLIF-SOFA grade $\geq 1$ were further categorized into the $\mathrm{AD}$ subgroup and the SS-induced ACLF subgroup.

The exclusion criteria were age $<18$ years, other causes of liver cirrhosis, pregnancy, alcoholism, trauma, hematological diseases, cancers, human immunodeficiency virus/acquired immunodeficiency syndrome (HIV/AIDS), autoimmune diseases, and taking steroidal/non-steroidal anti-inflammatory drugs or immunosuppressive agents during the previous 3 months.

All the LC and LC + SS patients received standard antiviral therapy, albumin, nutritional support and complication treatment and prevention measures as needed, and no patient had received a liver transplant. The treatment of SS was in accordance with the 2012 Surviving Sepsis Campaign guideline [18].

\section{Data collection}

For all the participants, demographic data and medical history were extracted during the enrollment. For the LC and the LC + SS group patients, relevant clinical data, auxiliary examination results and blood samples for flow cytometry analysis were obtained on day 1 of enrollment. For the LC + SS group patients, the Sequential Organ Failure Assessment (SOFA) score and the CLIF-SOFA score were calculated according to the data obtained on day 1 of enrollment. All the participants were followed up for 28 days to obtain all-cause mortality data.

\section{Flow cytometry}

$2 \mathrm{ml}$ of residual peripheral venous blood was obtained on admission from blood samples for clinical use, stored at 4 in a ethylenediamine tetraacetic acid (EDTA) tube and flow cytometry analysis was performed within 2 hours. PD-L1-PE (Cat\# 560176) and CD14-APC-H7 (Cat\# 560180) and their respective isotype controls recommended were purchased from BD Pharmingen (San Jose, CA, USA). $100 \mu$ l of whole blood sample was incubated with anti-CD14 and anti-PD-L1 antibodies for 20 minutes. Red blood cells were lysed. Anti-CD14 and anti-PD-L1 labeled cells and their isotypes labeled cells were obtained and run on a Gallios Flow Cytometer (Beckman Coulter, Inc.). Kaluza analysis version 1.5a (Beckman Coulter, Inc.) software was used for data analysis. Gating protocols of PD-L1 on CD14+ monocytes are shown in Figure 1.

\section{Statistical analysis}

The statistical analysis was performed with SPSS 22.0 software (IBM Corp., Armonk, NY, USA). Quantitative data were presented as medians $(\mathrm{M})$ and interquartile ranges. Qualitative data were reported as frequencies and per- 

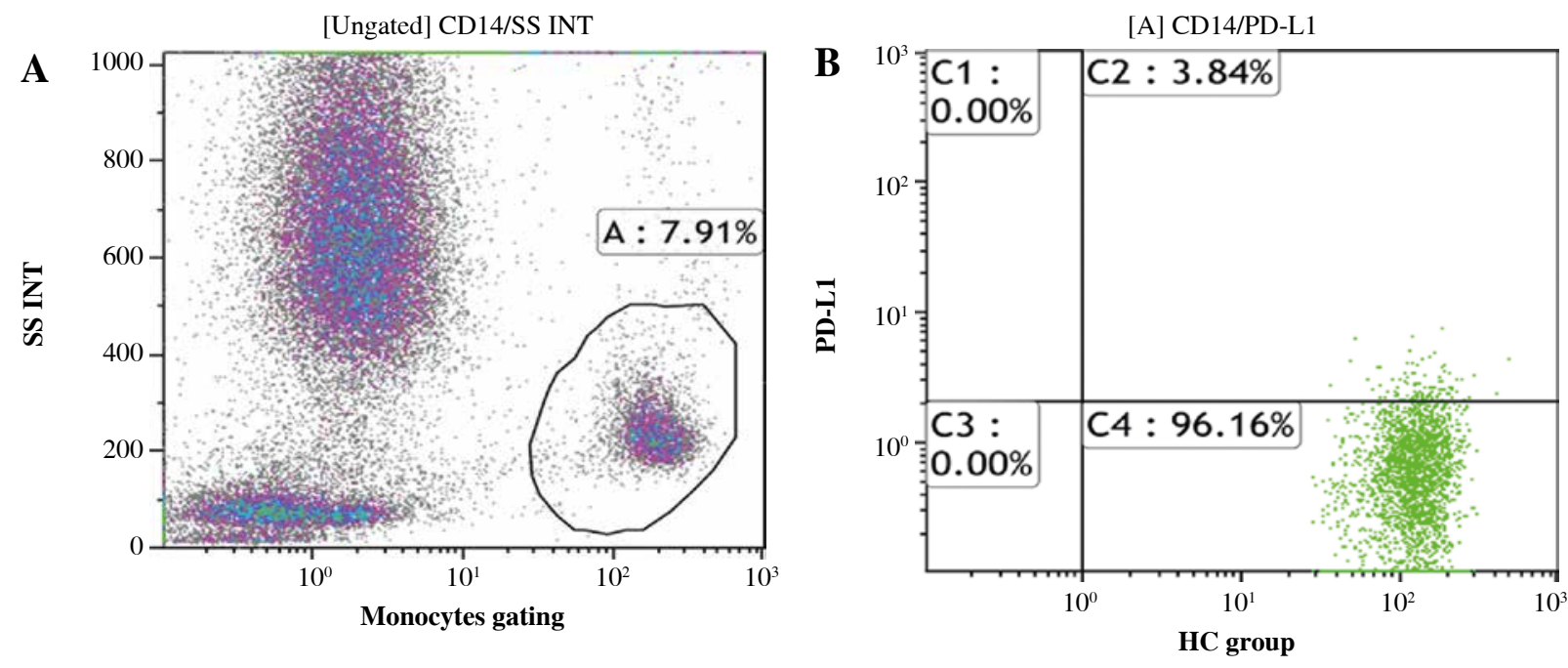

[A] CD14/PD-L1
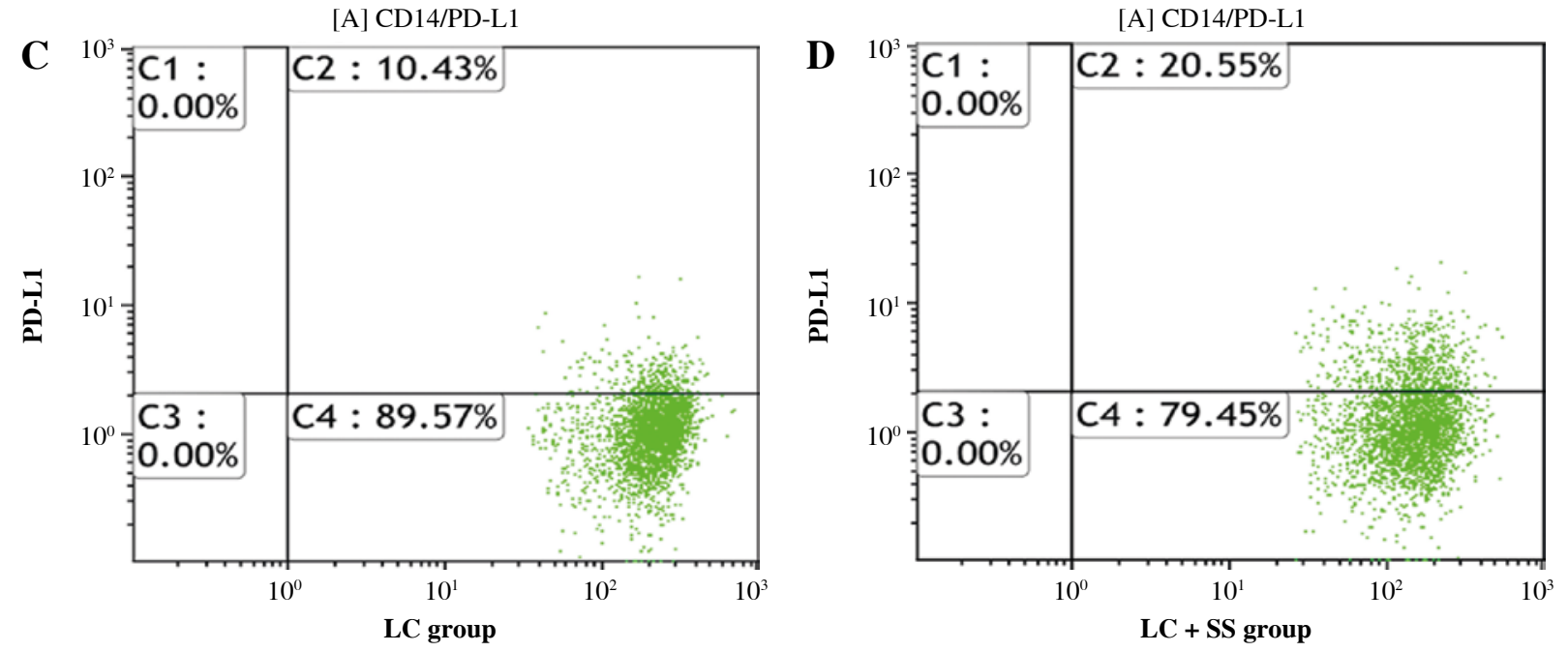

Fig. 1. Gating protocols of PD-L1 on CD14+ monocytes. A) CD14+ monocyte gating protocol. B-D) Monocytic PD-L1 expression in healthy control (HC), HBV-related liver cirrhosis (LC) and LC combined with severe sepsis (LC + SS) groups respectively. Gate A represents CD14+ monocytes. Gate C2 represents PD-L1 + CD14+ monocytes

centages. The comparisons of qualitative data and qualitative data were performed using the Mann-Whitney $U$ test and the chi-square test, respectively. Spearman's test was used to analyze the correlations between the CD14+ monocytic PD-L1 expression and the illness severity scores. A receiver operating characteristic (ROC) curve was used to analyze the value of different parameters for predicting 28-day mortality of SS-induced ACLF patients. We considered all statistical tests with a $p$ value $<0.05$ significant.

\section{Results}

\section{Participant characteristics}

A total of 117 eligible participants including $\mathrm{HC}(n=17)$, LC $(n=30)$ and LC + SS $(n=70)$ were enrolled in this prospective observational study from May 1, 2015 until September 30, 2016. No significant difference was observed with respect to age, sex and HBV viral load among the groups (both $p>0.05$ ). White blood cell (WBC) count was higher in the LC + SS group compared with the LC group $(p<0.001)$. Lymphocyte count was lower in the LC group compared with the HC group and the LC + SS group (both $p<0.001$ ). The positive culture rate was significantly higher in the LC $+\mathrm{SS}$ group compared with the LC group $(p<0.001)$. The baseline characteristics of the whole population are shown in Table 1.

After 28 days of follow-up, no death case was observed in the HC and the LC group. Out of $70 \mathrm{LC}+\mathrm{SS}$ patients, 59 were diagnosed with SS-induced ACLF. In the SS-induced ACLF subgroup, 37 patients survived. Age, sex, WBC count, lymphocyte count, HBV viral load and 
Table 1. Comparisons of baseline characteristics of healthy control (HC), HBV-related liver cirrhosis (LC) and HBV-related liver cirrhosis complicated with severe sepsis $(\mathrm{LC}+\mathrm{SS})$ groups

\begin{tabular}{lccc}
\hline Parameter & HC $(\boldsymbol{n}=\mathbf{1 7})$ & LC $(\boldsymbol{n}=\mathbf{3 0})$ & $\mathbf{L C}+\mathbf{S S}(\boldsymbol{n}=\mathbf{7 0})$ \\
\hline Age $($ years $)$ & $58(48-64)$ & $48(39-57)$ & $53(47-60)$ \\
\hline Male/total $(\%)$ & $10 / 17(58.8)$ & $21 / 30(70.0)$ & $49 / 70(70.0)$ \\
\hline WBC $\left(\times 10^{\%} / 1\right)$ & $5.82(4.89-7.73)$ & $3.46(2.55-5.84)$ & $8.84(5.60-12.67)^{*}$ \\
\hline Lymphocytes $\left(\times 10^{\%} / 1\right)$ & $2.43(1.98-3.16)$ & $1.01(0.76-1.39)^{*}$ & $0.83(0.54-1.21)^{* *}$ \\
\hline SOFA score & - & - & $10(8-12)$ \\
\hline CLIF-SOFA score & - & - & $13(10-15)$ \\
\hline PD-L1 percentage on CD14+ monocytes $(\%)$ & $3.10(1.40-6.56)$ & $11.91(2.24-28.62)^{*}$ & $20.05(8.56-41.90)^{* *}$ \\
\hline PD-L1 MFI on CD14+ monocytes $(\%)$ & $2.72(2.46-2.92)$ & $2.75(2.55-3.06)$ & $2.97(2.64-3.35)^{* *}$ \\
\hline HBV viral load log $(\mathrm{IU} / \mathrm{ml})(n=42)$ & - & $3.2(2-4.6)$ & $3.6(2.2-4.6)$ \\
\hline Culture results & - & $3(10.0)$ & $19(27.1)$ \\
\hline G+ cocci, $n(\%)$ & - & $2(6.7)$ & $11(15.7)$ \\
\hline G- bacilli, $n(\%)$ & - & $0(0)$ & $8(11.4)$ \\
\hline Mixed infection, $n(\%)$ & - & $0(0)$ & $5(7.1)$ \\
\hline Fungi, $n(\%)$ & - & $1(1.4)$ \\
\hline Anaerobes, $n(\%)$ & - & & \\
\hline
\end{tabular}

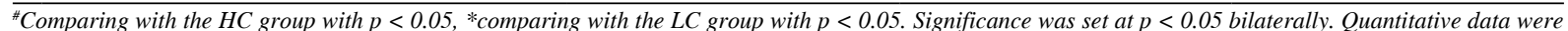
presented as medians $(M)$ and interquartile ranges. Qualitative data were reported as frequencies and percentages.

culture results showed no significant difference between survivors and non-survivors (all $p>0.05$ ). Intra-abdomen was the most frequent site of infection in both the survivors (23/37) and the non-survivors (17/22). The baseline data of SS-induced ACLF patients are shown in Table 2.

\section{PD-L1 expression on monocytes among the $\mathrm{HC}$, $\mathrm{LC}$ and $\mathrm{LC}+\mathrm{SS}$ groups}

The monocytic PD-L1 percentage was higher in the LC + SS group compared with the LC $(p=0.046)$ and the HC groups $(p<0.001)$ and lower in the HC group compared with the LC group $(p=0.018)$. The monocytic PD-L1 mean fluorescence intensity (MFI) was higher in the LC + SS group compared with the LC $(p=0.046)$ and the HC $(p=0.009)$ groups. Comparisons of PD-L1 expression on CD14+ monocytes among the HC, LC and LC + SS groups are shown in Table 1.

The percentage and MFI of monocytic PD-L1 were positively correlated in both the whole cohort $(r=0.660$, $p<0.001)$ and the LC + SS group $(r=0.756, p<0.001)$. The MFI of PD-L1 on CD14+ monocytes represents the average quantity of PD-L1 molecules expressed on the surface of a single monocyte. The close correlations confirmed the reliability of the experiment. Figure 2A, B represents the relationships between the percentage and the MFI of PD-L1 on CD14+ monocytes in the whole cohort and in the LC $+\mathrm{SS}$ group respectively.

\section{Correlations between monocytic PD-L1 expression} and illness severity scores of $\mathbf{L C}+\mathrm{SS}$ patients

The monocytic PD-L1 percentage was positively correlated with the SOFA score $(r=0.328, p=0.006)$ and the CLIF-SOFA score $(r=0.389, p=0.001)$. The monocytic PD-L1 MFI was also positively correlated with the CLIF-SOFA score $(r=0.324, p=0.006)$. The correlations between the expression of PD-L1 on CD14+ monocytes and illness severity scores are shown Figure 2C-F.

\section{Comparisons between survivors and non- survivors in the SS-induced HBV-ACLF subgroup during a 28-day follow-up}

According to the CLIF-SOFA grading system, LC + SS patients were divided into 4 grades (grade 0-3). 11 patients $(15.7 \%)$ were categorized in the AD subgroup and 59 patients $(84.3 \%)$ in the SS-induced HBV-ACLF subgroup. All the deaths cases (22 cases) were reported in the SS-induced HBV-ACLF subgroup. The monocytic PD-L1 percentage $(p=0.004)$ and MFI $(p=0.008)$, the SOFA score $(p<0.001)$, and the CLIF-SOFA score $(p<0.001)$ were significantly higher in the non-survivors compared with the survivors. The patients with CLIF-SOFA grade 3 exhibited a significantly higher mortality rate compared with the patients with CLIF-SOFA grade $1+2(p<0.001)$. The comparisons of PD-L1 expression on CD14+ monocytes between the survivors and non-survivors are shown in Table 2. 
Table 2. Baseline data of survivors vs. non-survivors in the SS-induced HBV-ACLF subgroup

\begin{tabular}{|c|c|c|c|}
\hline Parameter & Survivors $(n=37)$ & Non-survivors $(n=22)$ & $P$-value \\
\hline Age (years) & $53(48-59)$ & $56(48-65)$ & 0.147 \\
\hline Male, $n(\%)$ & $25(67.6)$ & $15(68.2)$ & 0.961 \\
\hline $\mathrm{WBC}\left(\times 10^{9} / 1\right)$ & $7.77(4.58-11.52)$ & $10.54(6.38-14.89)$ & 0.163 \\
\hline Lymphocytes $\left(\times 10^{9} / 1\right)$ & $0.81(0.38-1.26)$ & $0.70(0.60-1.24)$ & 0.689 \\
\hline SOFA score & $9(7-11)$ & $13.5(11.8-15)$ & $<0.001^{\dagger}$ \\
\hline CLIF-SOFA score & $11(9-13)$ & $16.5(13-17.3)$ & $<0.001^{\#}$ \\
\hline PD-L1 percentage on CD14+ monocytes (\%) & $13.63(5.33-29.43)$ & $36.79(15.41-47.73)$ & $0.004^{\#}$ \\
\hline PD-L1 MFI on CD14+ monocytes (\%) & $2.80(2.60-3.26)$ & $3.24(2.81-3.88)$ & $0.008^{\#}$ \\
\hline HBV viral load $\log (\mathrm{IU} / \mathrm{ml})(n=42)$ & $3.7(2-4.7)$ & $3.9(3.3-4.5)$ & 0.490 \\
\hline Culture results & & & 0.351 \\
\hline $\mathrm{G}+\operatorname{cocci}, n(\%)$ & $13(35.1)$ & $4(18.2)$ & - \\
\hline G- bacilli, $n(\%)$ & $5(13.5)$ & $3(13.6)$ & - \\
\hline Mixed infection, $n(\%)$ & $4(10.8)$ & $2(9.1)$ & - \\
\hline Fungi, $n(\%)$ & $1(2.7)$ & $3(13.6)$ & - \\
\hline Anaerobes, $n(\%)$ & $0(0)$ & $1(4.5)$ & - \\
\hline Site of infection & & & 0.653 \\
\hline Lung, $n(\%)$ & $10(27.0)$ & $3(13.6)$ & - \\
\hline Intra abdomen, $n(\%)$ & $23(62.1)$ & $17(77.3)$ & - \\
\hline GI tract, $n(\%)$ & $2(5.4)$ & $1(4.5)$ & - \\
\hline Urinary tract, $n(\%)$ & $2(5.4)$ & $1(4.5)$ & - \\
\hline
\end{tabular}

\section{Monocytic PD-L1 expression for predicting 28-day mortality of the SS-induced HBV-ACLF patients}

In the receiver operating characteristic (ROC) curve for predicting 28-day mortality of the SS-induced HBV-ACLF patients (Fig. 3), the area under the curve (AUC) of the monocytic PD-L1 percentage, the monocytic PD-L1 MFI, the SOFA score and the CLIF-SOFA score were 0.727 $(p=0.004), 0.709(p=0.008), 0.924(p<0.001)$ and 0.813 $(p<0.001)$ respectively.

In the ROC curves, according to the maximization of Youden index principle, $12.35 \%$ (sensitivity 0.909 , specificity 0.459 ) and 2.67 (sensitivity 0.909 , specificity 0.432 ) were identified the optimal cutoff points for monocytic PD-L1 percentage and MFI respectively. Patients above the PD-L1 percentage cutoff points exhibited a significantly higher 28-day mortality $(\mathrm{OR}=8.50$, 95\% CI: $1.73-41.72, p=0.004)$, SOFA score $(p=0.044)$ and CLIF-SOFA score $(p=0.010)$ compared with patients under the cutoff point. Patients above the PD-L1 MFI cutoff point also exhibited significantly higher risk of death (OR $=7.62,95 \%$ CI: $1.56-37.45, p=0.008)$, SOFA score $(p=0.024)$ and CLIF-SOFA score $(p=0.012)$.

\section{Discussion}

Immunosuppression plays an important role in bacterial infections in cirrhotic patients [4]. PD-L1 overexpression contributes to sepsis-associated immunosuppression. PD-1:PD-L1 pathway blocking restores T cell function and improves sepsis prognosis [19-22]. PD-1 and PD-L1 overexpression were also found in liver cirrhosis patients $[23,24]$. Anti-PD-1 therapy in a PD-1 overexpressed CHB mouse model exhibited improved prognosis [25] while anti-PD-L1 agents increased peripheral blood mononuclear cell proliferation and enhanced interferon $\gamma$ $(\mathrm{INF}-\gamma)$ production $[26,27]$. In a sepsis model, PD-L1 up-regulation was associated with monocytic dysfunction and PD-L1 blocking decreased the secretion of interleukin (IL)-10, and increased the secretion of tumor necrosis factor $\alpha$ (TNF- $\alpha)$ and IL- 6 , which in turn increased bacterial clearance [14]. These studies imply that monocytic PD-L1 over-expression may be superimposed in LC + SS patients, especially in SS-induced ACLF patients, and this population may further benefit from PD-L1 blocking agents by reversing PD-1:PD-L1 pathway related immunosuppression.

In the present study, monocytic PD-L1 was over-expressed in LC patients and when complicated with SS, 


\section{A}

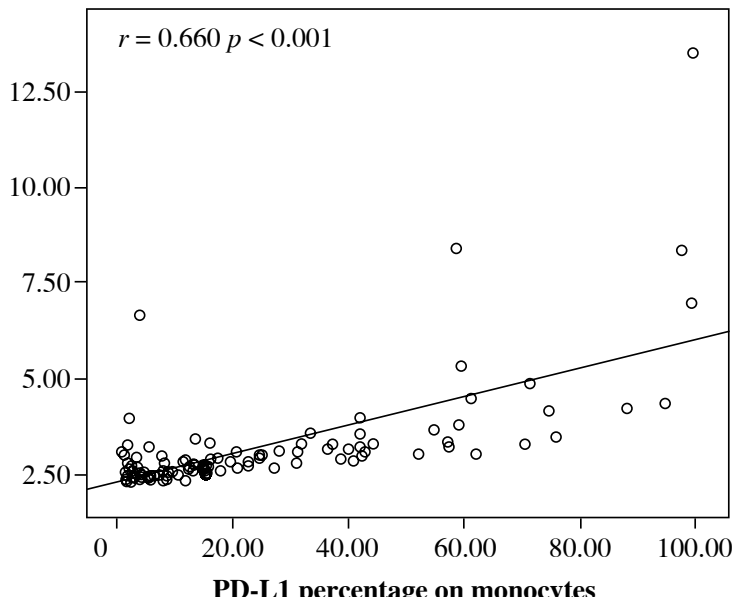

PD-L1 percentage on monocytes

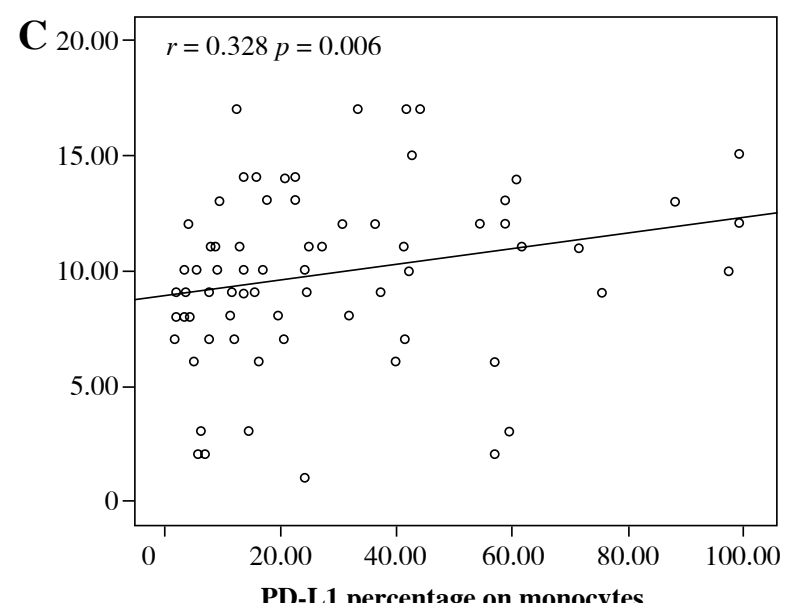

PD-L1 percentage on monocytes

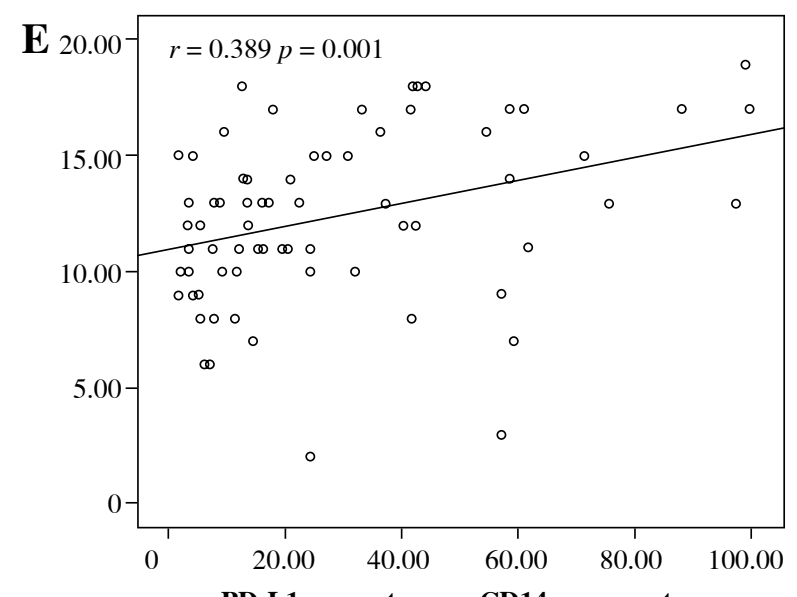

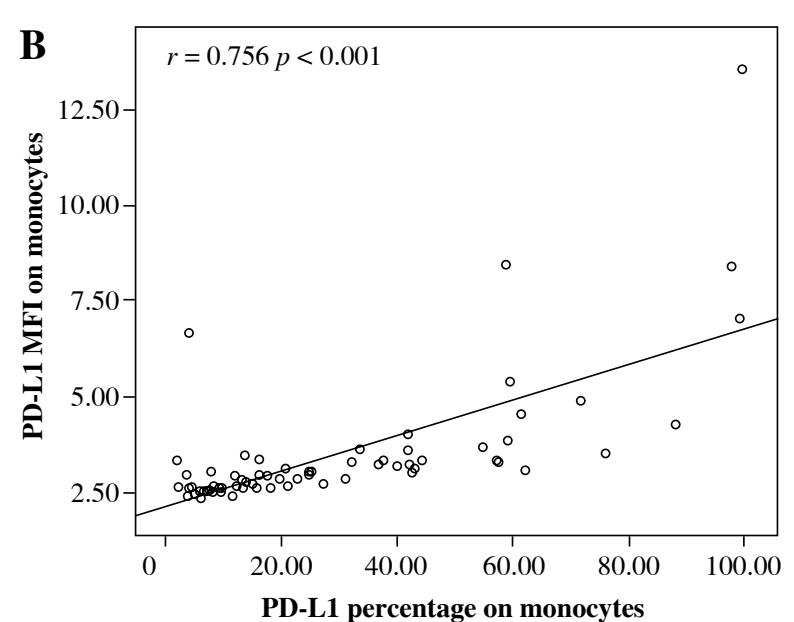
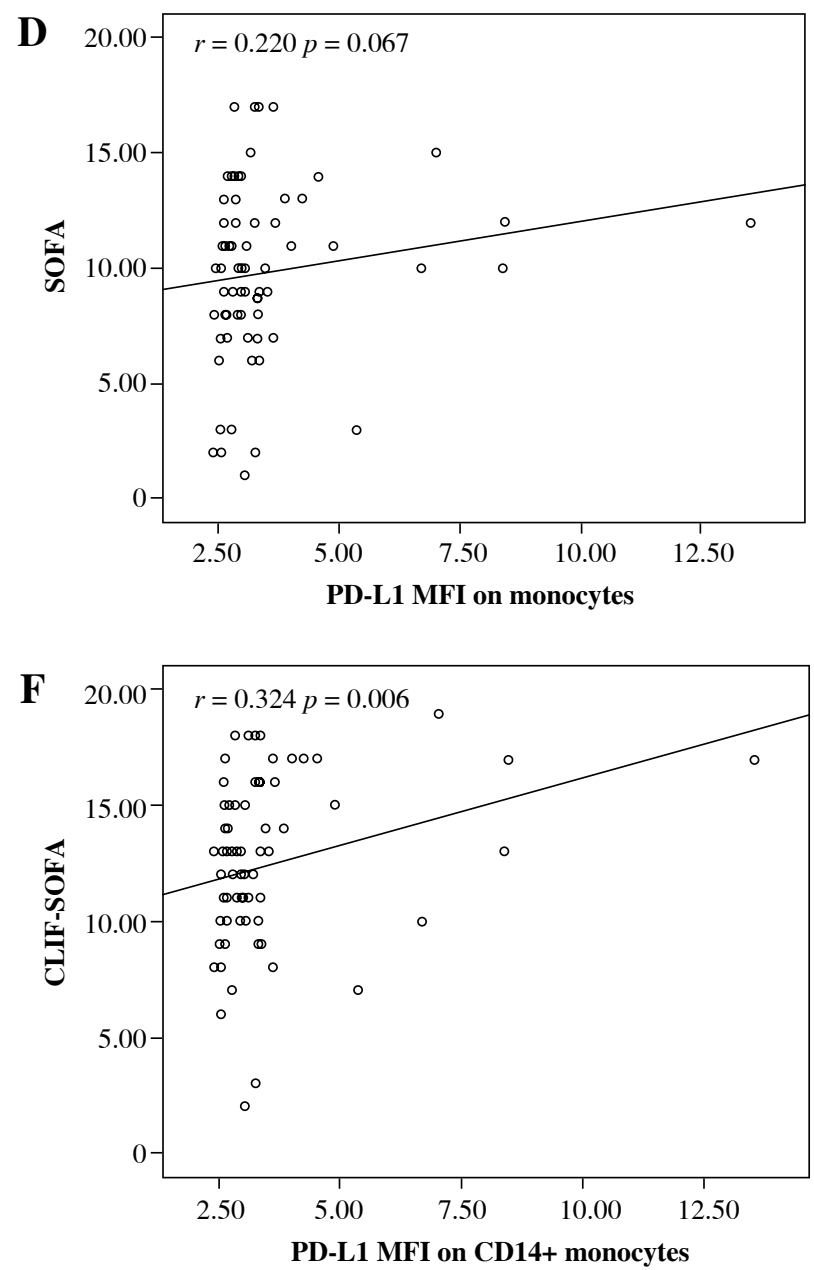

SOFA - Sequential Organ Failure Assessment, CLIF-SOFA - Chronic Liver Failure-Sequential Organ Failure Assessment, PD-L1 - programmed death ligand-1, $M F I$ - mean fluorescence intensity

Fig. 2. A, B) The correlations between CD14+ monocytic PD-L1 percentage and MFI in the whole population and in the LC + SS group respectively. C-F) The correlations between PD-L1 expression (percentage and MFI) on CD14+ monocytes and illness severity scores (SOFA score and CLIF-SOFA score) 
monocytic PD-L1 expression further increased. It is reasonable to infer that in LC $+\mathrm{SS}$ patients, especially LC-induced HBV-ACLF patients, increased expression of monocytic PD-L1 may be involved in the deteriorated overall immunosuppression and contribute to increased mortality. However, this hypothesis needs to be further investigated.

In a previously study by our partners, the investigators examined PD-L1 expression on CD14+ monocytes in sepsis patients and healthy controls using the same protocol [15]. It was found that the monocytic PD-L1 expression rates were $18.5 \%, 21.5 \%$ and $21.0 \%$ in the control group, severe sepsis group and septic shock group, respectively. The study enrolled a relatively large population ( 29 healthy control, 59 severe septic patients, and 76 septic shock patients). Severe sepsis or septic shock patients exhibited only a 20-25\% increase of PD-L1 expression on CD14+ macrophages compared with the control group. In the present study, however, we observed a 3-4 fold and 6-7 fold increase of monocytic PD-L1 expression in the LC group and LC + SS group compared with the healthy control group, respectively, Although the baseline characteristics and sampling time differed between our study and the previous study [15], it is speculated that PD-L1 expression on CD14+ monocytes may be significantly higher in LC + SS patients compared with the SS patients. It is important to add an additional SS group to examine whether the elevated monocytic PD-L1 expression was due to the simple summation effect of LC and SS, or the intensification of interactions between LC and SS.

Biomarkers for predicting the prognosis of septic patients has been a clinical focus. Traditional biomarkers were developed largely based on the theories of cytokine storm and pro-inflammatory responses [5]. However, it was observed that immunosuppression may be present from the very start of sepsis and determined the fate of sepsis [28]. Previously, PD-L1 had been proved a prognostic marker of both septic shock and ACLF $[15,17]$. Here, we examined the prognostic value of this parameter in the SS-induced HBV-ACLF population. We found that monocytic PD-L1 expression was positively correlated with clinical severity scores in the LC + SS group, in which more than $80 \%$ of patients were diagnosed with HBV-ACLF. Also, all the death cases occurred in the patients with initial CLIF-SOFA grade $>0$, indicating that SS-induced HBV-ACLF was responsible for the 28-day mortality of $\mathrm{LC}+\mathrm{SS}$ and AD generally led to a satisfactory prognosis. In the SS-induced HBV-ACLF subgroup, both monocytic PDL-1 percentage and MFI exhibited excellent predictive values. However, the value of this parameters for predicting the clinical outcome of this specific population needs to be further evaluated in a large-scale population.

The following limitations need to be considered: firstly, this was a preliminary study with a relatively small

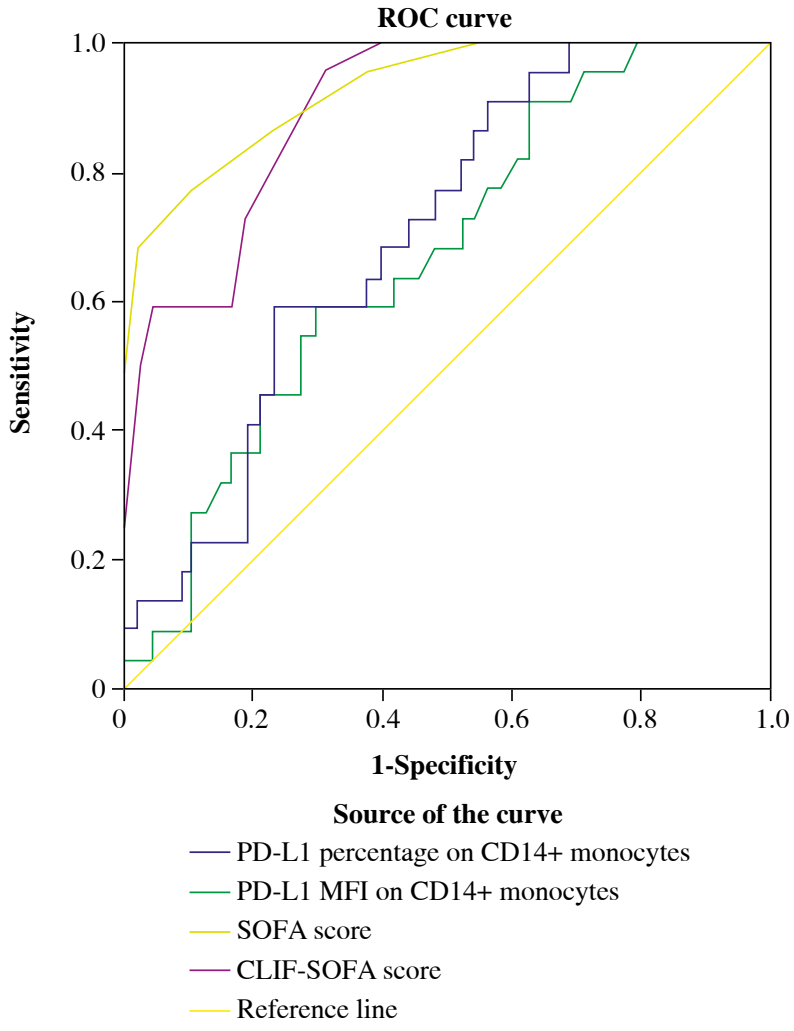

SOFA - Sequential Organ Failure Assessment, CLIF-SOFA - Chronic Liver Failure-Sequential Organ Failure Assessment, PD-L1 - programmed death ligand-1, MFI-mean fluorescence intensity

Fig. 3. The receiver operating characteristic (ROC) curve for predicting 28-day mortality of SS-induced ACLF patients

sample size. The predictive value of monocytic PD-L1 expression in SS-induced HBV-ACLF needs further evaluation. Secondly, this study only examined decompensated HBV-related liver cirrhotic patients, which limits the generalization of conclusions. Thirdly, peripheral PD-L1 monocytes could not represent intra-hepatic conditions. Fourthly, this pilot observational study only revealed an additive effect of LC and SS on monocytic PD-L1 expression during admission. It is important to add an SS group to examine the cause of monocytic PD-L1 expression elevation in $\mathrm{LC}+\mathrm{SS}$ patients and examine monocytic PD-L1 expression at different time points in the future study. Lastly, as the results are markedly influenced by the internal (enrolled population and experimental design) and external factors (experimental instruments, equipment, reagents, experimenters etc.), a large-scale study with detailed subgroup analysis is mandatory to confirm the results.

\section{Conclusions}

Severe sepsis exhibits an superimposed effect on monocytic PD-L1 up-regulation on the basis of liver cir- 
rhosis, and monocytic PD-L1 expression predicts 28-day mortality of SS-induced HBV-ACLF.

\section{Acknowledgements}

We would like to thank the Emergency Department staff in Beijing Di-tan Hospital and the Biochemistry Laboratory staff at the Medical Research Center of Beijing Chao-yang Hospital. There was no funding.

\section{The authors declare no conflict of interest.}

\section{References}

1. Arvaniti V, D'Amico G, Fede G, et al. (2010): Infections in patients with cirrhosis increase mortality four-fold and should be used in determining prognosis. Gastroenterology 139: 1246-1256.

2. Moreau R, Jalan R, Gines P, et al. (2013): CANONIC Study Investigators of the EASL-CLIF Consortium. Acute-onchronic liver failure is a distinct syndrome that develops in patients with acute decompensation of cirrhosis. Gastroenterology 144: 1426-1437.

3. Wang FS, Fan JG, Zhang Z, et al. (2014): The global burden of liver disease: the major impact of China. Hepatology 60: 2099-2108.

4. Azeem A, Ka CS, Daqing M (2017): Acute-on-chronic liver failure: recent update. J Biomed Res 31: 283-300.

5. Philips CA, Sarin SK (2016): Sepsis in cirrhosis: emerging concepts in pathogenesis, diagnosis and management. Hepatol Int 10: 871-882.

6. Sun YY, Li XF, Meng XM, et al. (2017): Macrophage phenotype in liver injury and repair. Scand J Immunol 85: 166-174.

7. Triantafyllou E, Woollard KJ, McPhail MJW, et al. (2018): The role of monocytes and macrophages in acute and acuteon-chronic liver failure. Front Immunol 9: 2948.

8. Cavaillon JM, Adib-Conquy M (2005): Monocytes/macrophages and sepsis. Crit Care Med 33 (12 Suppl): S506-509.

9. Barber DL, Wherry EJ, Masopust D, et al. (2006): Restoring function in exhausted CD8 T cells during chronic viral infection. Nature 439: 682-687.

10. Keir ME, Butte MJ, Freeman GJ, et al. (2008): PD-1 and its ligands in tolerance and immunity. Annu Rev Immunol 26: 677-704.

11. Dong Y, Sun Q, Zhang X (2016): PD-1 and its ligands are important immune checkpoints in cancer. Oncotarget 8: 2171-2186.

12. Yao S, Chen L (2014): PD-1 as an immune modulatory receptor. Cancer J 20: 262-264.

13. Francisco LM, Salinas VH, Brown KE, et al. (2009): PDL1 regulates the development, maintenance, and function of induced regulatory T cells. J Exp Med 206: 3015-3029.

14. Zhang Y, Zhou Y, Lou J, et al. (2010): PD-L1 blockade improves survival in experimental sepsis by inhibiting lymphocyte apoptosis and reversing monocyte dysfunction. Crit Care 14: R220.

15. Shao R, Fang Y, Yu H, et al. (2016): Monocyte programmed death ligand-1 expression after 3-4 days of sepsis is associated with risk stratification and mortality in septic patients: a prospective cohort study. Crit Care 20: 124.
16. Zhang M, Wang FL, Zhu JY, et al. (2013): Liver myofibroblasts regulate the phenotype and function of monocytes through soluble factors in cirrhosis. Exp Ther Med 5: 143149.

17. Xie Z, Chen Y, Zhao S, et al. (2009): Intrahepatic PD-1/ PD-L1 up-regulation closely correlates with inflammation and virus replication in patients with chronic HBV infection. Immunol Invest 38: 624-638.

18. Dellinger RP, Levy MM, Rhodes A, et al.; Surviving Sepsis Campaign Guidelines Committee including The Pediatric Subgroup (2013): Surviving Sepsis Campaign: international guidelines for management of severe sepsis and septic shock. Intensive Care Med 39: 165-228.

19. Chang KC, Burnham CA, Compton SM, et al. (2013): Blockade of the negative co-stimulatory molecules PD-1 and CTLA-4 improves survival in primary and secondary fungal sepsis. Crit Care 17: R85.

20. Wang JF, Li JB, Zhao YJ, et al. (2015): Up-regulation of programmed cell death 1 ligand 1 on neutrophils may be involved in sepsis-induced immunosuppression: an animal study and a prospective case-control study. Anesthesiology 122: 852-863.

21. Shindo Y, McDonough JS, Chang KC, et al. (2017): AntiPD-L1 peptide improves survival in sepsis. J Surg Res 208: 33-39.

22. West EE, Jin HT, Rasheed AU, et al. (2013): PD-L1 blockade synergizes with IL-2 therapy in reinvigorating exhausted T cells. J Clin Invest 123: 2604-2615.

23. Xu P, Chen YJ, Chen H, et al. (2014): The expression of programmed death-1 in circulating CD4+ and CD8+ T cells during hepatitis $\mathrm{B}$ virus infection progression and its correlation with clinical baseline characteristics. Gut Liver 8: 186-195.

24. Zhang M, Wang FL, Zhu JY, et al. (2013): Liver myofibroblasts regulate the phenotype and function of monocytes through soluble factors in cirrhosis. Exp Ther Med 5: 143-149.

25. Tzeng HT, Tsai HF, Liao HJ, et al. (2012): PD-1 blockade reverses immune dysfunction and hepatitis $\mathrm{B}$ viral persistence in a mouse animal model. PLoS One 7: e39179.

26. Peng G, Luo B, Li J, et al. (2011): Hepatitis B e-antigen persistency is associated with the properties of $\mathrm{HBV}$-specific CD8 T cells in CHB patients. J Clin Immunol 31: 195-204.

27. Fisicaro P, Valdatta C, Massari M, et al. (2012): Combined blockade of programmed death-1 and activation of CD137 increase responses of human liver $\mathrm{T}$ cells against $\mathrm{HBV}$, but not HCV. Gastroenterology 143: 1576-1585.e4.

28. Yadav H, Cartin-Ceba R (2016): Balance between hyperinflammation and immunosuppression in sepsis. Semin Respir Crit Care Med 37: 42-50. 